\title{
A PLL-Based Online Estimation of Induction Motor Consumption Without Electrical Measurement
}

\author{
Thierry Doget *, Erik Etien, Laurent Rambault $D$ and Sébastien Cauet \\ LIAS, Université de Poitiers, 86000 Poitiers, France; erik.etien@univ-poitiers.fr (E.E.); \\ laurent.rambault@univ-poitiers.fr (L.R.); sebastien.cauet@univ-poitiers.fr (S.C.) \\ * Correspondence: thierry.doget@univ-poitiers.fr
}

Received: 6 October 2018; Accepted: 17 April 2019; Published: 25 April 2019

\begin{abstract}
This work is supported by a company wishing to develop new products in the field of energy monitoring in industry. It concerns the real-time estimation of the electrical consumption of an asynchronous motor without electrical measurement. The challenge consists of estimating the characteristic quantities of the motor (speed, torque, powers, efficiency) with only one vibratory measurement, information on the nameplate and commercial documentation available online. To obtain a real-time estimate, traditional FFT analysis is replaced by a PLL initially designed for power grid analysis. So, the second challenge is to modify this PLL for use with vibratory measurement characterized by a low signal-to-noise ratio, amplitude variations and a non-stationary behavior. A complete design and experimental tests are presented to validate the proposed approach.
\end{abstract}

Keywords: electrical power estimation; induction motor; vibrations analysis; PLL

\section{Introduction}

This study concerns the control of electricity consumption in industry. In this sector, electrical motors are used in multiple uses (lifting, ventilation, air conditioning, pumping, etc.) and represent an important part of the final electricity consumption. These uses are the subject of particular attention during the processes of estimation of consumption and energy audit. It is clear that electrical measurements (currents, voltages) are the most direct and precise way to estimate the consumption of loads, but, in practice, they are subjected to several constraints :

- conventional energy measurement requires the access to electrical quantities (current, voltage) either as close as possible to the system or in the electrical cabinet where the system is connected;

- the electrical line must feed only the system concerned and not a group consisting of several loads;

- the electrical distribution of the entire site must be perfectly known (electrical drawings are not even available).

In the context of the energy audit, two additional constraints are added:

- the measuring instruments shall be installed for a period representative of the intended use. Ideally, annual consumption is sought. A measure of consumption over too short a period requires an estimate of the annual operating time in an empirical way, which leads to great uncertainty in the calculation of the annual energy consumption;

- $\quad$ in the case of large sites, the number of motors can be large, which multiplies the measurement points and can increase the cost of the energy audit process in a prohibitive way.

The objective of this study is to design an electrical energy meter for electrical machines without current and voltage sensors. This strategy makes it possible to overcome the constraints linked to the 
fine knowledge of the network and to deal with the cases where the uses are grouped on the same departure. It will thus, be able to be deployed over a large number of uses and be left to remain for a sufficiently long period of time to obtain a significant estimate of the energy consumed. The method is constructed from a single sensor providing :

- $\quad$ the running time of the use $(T)$;

- $\quad$ the measurement of the motor speed allowing the electrical power estimation $\left(P_{e}\right)$.

The electrical energy consumed $\left(W_{e}\right)$ will then be deduced from the relation :

$$
W_{e}=P_{e} \times T
$$

In a conventional auditing process, electrical power is measured accurately but over a relatively short time. On the other hand, the annual operating time is estimated approximately, which leads to an unreliable calculated energy. The strategy proposed in our paper proposes to do the reverse, that is to say to have a very precise time estimate by conceding a loss of precision on the power measurement. To do this, the vibrations emitted by the motor are used as source of information. The velocity and the electrical frequency can be estimated by spectral analysis. Electrical power can then be calculated from motor models. Traditionally, the signal spectrum is obtained using an FFT algorithm [1-3] or Time-frequency methods when signals are non-stationnary [4]. As it will be seen in the following, the motor models require a high precision on the frequency measurement. The sought frequencies are relatively low, of the order of $50 \mathrm{~Hz}$ for a motor running at $3000 \mathrm{rpm}$. This involves long recording times incompatible with the specifications of an energy meter. As an example, our system should be able to provide an estimate of electrical power every second. Therefore, we chose to use another technique based on PLL.

Phase Lock Loops (PLLs) are often used in all types of connected systems because they are fast and accurate [5-7]. The basic structure of a PLL consists of three fundamental blocks: a phase detector (PD), a loop filter (LF) and a voltage controlled oscillator (VCO). A simple multiplier can be commonly used for PDs but it generates a harmonic of high frequency and amplitude which limits the bandwidth of the PLL and therefore increases its response time. A PD based on quadrature signals can solve the harmonic problem [8,9]. This type of system uses an Orthogonal Signal Generator (OSG) to generate two-phase orthogonal signals applied to a Park transformation to obtain high accuracy on the phase error. In $[10,11]$, authors present various PLLs based on the OSG. Detailed comparisons were made from the following PLG-GSOs: Delay-PLL, Der-PLL, Park-PLL, SOGI-PLL, DOEC-PLL, VTD-PLL, CCF-PLL and TPFA-PLL algorithms to obtain vision of their advantages and disadvantages. The comparative study is carried out under various network perturbations such as voltage failures, phase and frequency jumps, but also in the presence of harmonics and offset Dc voltage and noise at the input of the PLLs. The reference PLLs are built on the SOGI topology, which provides good performance [12-14]. They can be combined with a frequency-locked loop (FLL) to improve their performance on the particular extraction of a frequency component. From this work, it follows that the SOGI-PLL presents some problems related to its dynamic response during the rapid change of the central frequency and can under oscillate under certain conditions. Ref. [15] proposes an original PLL based on derived elements (DE) and an OSG constructed from two DEs with the same parameterization. Appropriate parameterization makes it possible to generate orthogonal signals without the risk of oscillation. The results of the comparison between the SOGI-PLL and the DE-PLL show significant performances of the latter in terms of improvements in system dynamics and PLL accuracy while maintaining robust performance at network disruptions.

These PLLs are generally used to estimate frequencies from electrical signals (currents or voltages) measured on the network. In this particular case, the frequency is relatively stable and not very noisy. In this article we show that it is possible to adapt this type of PLL to a vibratory signal adding some improvements. 
In the Section 2, the method for estimating electric power is presented. Emphasis will be placed on the accuracy required for estimating the rotational speed due to the sensitivity of the models used with respect to the speed of rotation. In Section 3, the estimation of the rotational speed based on the PLL will be detailed. It will be shown that a real-time estimate can be obtained with sufficient accuracy for our application. The experimental tests validating the proposed method will be presented in Section 4.

\section{Principle of Electrical Power Estimation}

\subsection{Introduction}

The principle of electrical power estimation is given in Figure 1.

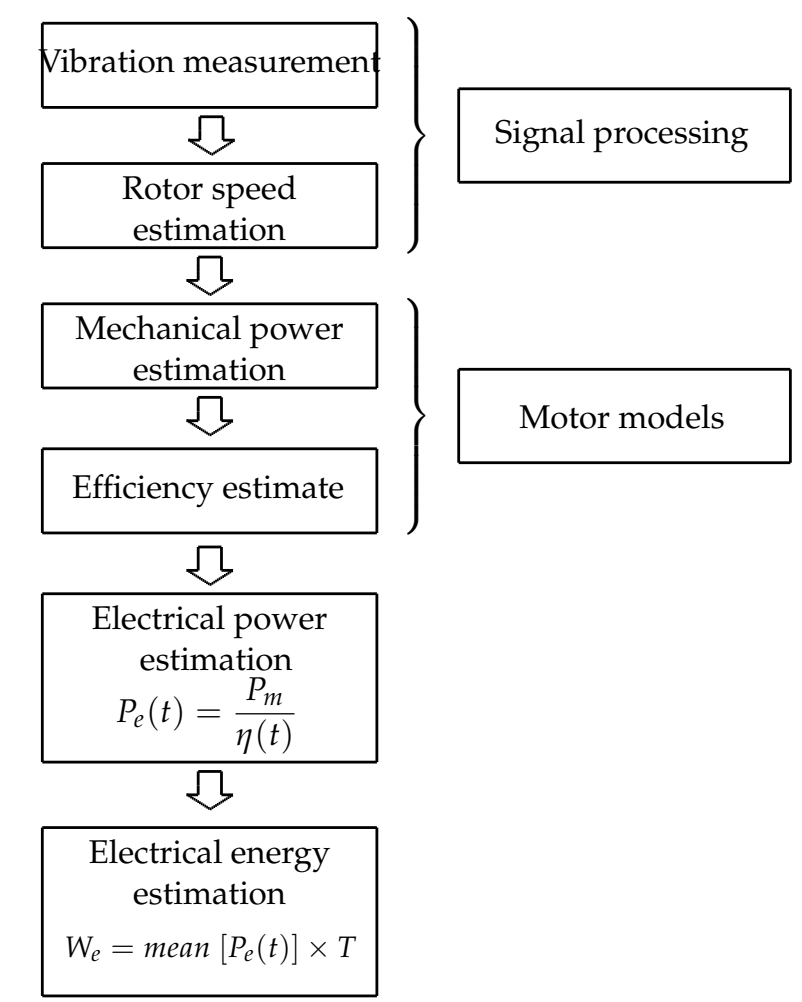

Figure 1. Principle of electrical energy estimation.

In Section 2.2, we recall the spectral content of the vibratory signal and show that the speed rotation can be deduced from this spectrum. The rotational speed estimation makes it possible to determine the mechanical power and the efficiency from the following motor characteristics :

- $\quad P_{m}=f\left(N_{m}\right)$ : mechanical power $\left(P_{m}\right)$ as a function of the speed rotational $\left(N_{m}\right)$;

- $\quad \eta=h\left(P_{m}\right)$ : efficiency as a function of mechanical power.

This data can be provided by the motor manufacturer. If these data are not available, the characteristic $P_{m}=f\left(N_{m}\right)$ can be approximated by the slip method. In the same way, the efficiency curve can be approximated from models, database or standards [16-19]. One important point is the sensitivity of these two models to the accuracy of the speed estimation. Indeed, for an asynchronous motor, the characteristic $P_{m}=f(N)$ can be approximated by a quasi-vertical line. Therefore, a small error on the speed estimate can lead to an erroneous estimate of the mechanical power. This problem will be discussed in the Section 2.3.3. 


\subsection{Vibration Model}

Generally, due to motor imperfections, a vibrations measurement provides a signal that can contain harmonic components at specific frequencies as motor speed, electrical frequency and possible electrical or mechanical faults.

This information is accessible by spectral analysis and a precise location of particular spectral components [20]:

$$
\begin{array}{r}
f_{v 1}=g S_{r} f_{r m}+k_{1} f_{e} \\
f_{v 2}=g S_{r} f_{r m}+k_{1} f_{e} \pm f_{r m} \\
f_{v 3}=g S_{r} f_{r m}+k_{2} f_{e}
\end{array}
$$

With:

$f_{v 1}$ : frequency linked the radial force due to the harmonics of rotor slots.

$f_{v 2}$ : frequency due to the eccentricity of the rotor.

$f_{v 3}$ : frequency due to saturation of the rotor.

$g$ : constant equal to $0, \pm 1, \pm 2 \ldots$

$S_{r}$ : Number of rotor slots.

$f_{r m}$ : rotation frequency of the rotor.

$f_{e}$ : electrical supply frequency.

$k_{1}$ : constant equal to 0 or 2 .

$k_{2}$ : constant equal to 2 or 4 .

We note that by fixing $g=0$ and $k_{1}=0$ in the second expression of (2), one obtains $f_{v 2}= \pm f_{r m}$. Consequently, the spectrum of the vibratory signal of a motor having an eccentricity defect contains the rotational speed information. It should be noted that the information sought is not the amplitude at the frequency component at $f=f_{r m}$ but the exact value of $f_{r m}$. Thus, even in the presence of a slight lack of eccentricity, the location of this frequency remains possible. It should also be noted that the information related to the electrical supply frequency is also available $\left(g=0, k_{1}=2\right)$. Let us verify this model on experimental measurements. The test motor is a $3 \mathrm{~kW} / 1500 \mathrm{rpm}$ induction motor whose characteristics are given in Figure 2.

\begin{tabular}{|l|l|}
\hline$N_{m_{N}}($ rpm $)$ & 1435 \\
\hline$P_{m_{N}}(W)$ & 3000 \\
\hline$U_{N} / V_{N}(V)$ & $230 / 133$ \\
\hline$I_{N} / J_{N}(A)$ & $11 / 18.9$ \\
\hline$D P F_{N}$ & 0.84 \\
\hline
\end{tabular}

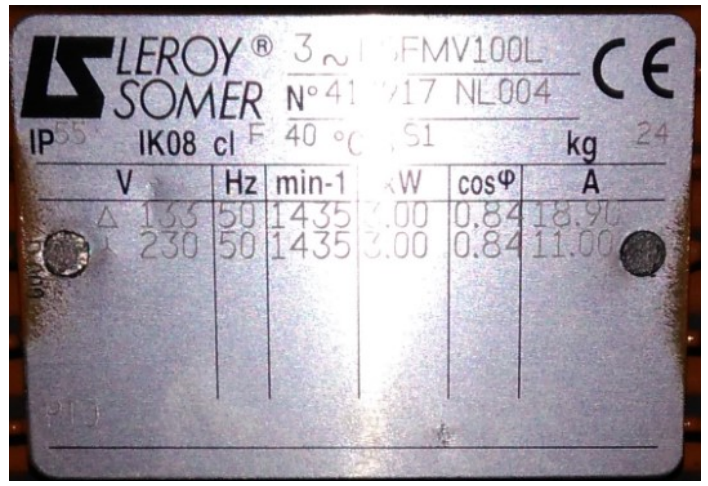

Figure 2. Motor informations.

From these information, the nominal electrical power can calculated $P_{e_{N}}=U_{N} I_{N} \sqrt{3} D P F_{N}=3681 \mathrm{~W}$ and the nominal efficiency $\eta_{N}=P_{m_{N}} / P_{e_{N}}=0.815$.

This motor is connected to a generator controllable in torque. A vibratory sensor is stuck on the asynchronous motor. Furthermore, the electrical power $\left(P_{e}\right)$ is directly measured at the motor input. It is the only direct measurement available on this test bench. The signals recording is performed by a dSPACE-DS1104 card and the acquisition is made in MATLAB environment. The sampling frequency is $F_{s}=10 \mathrm{kHz}$ and the duration of the recording is $D=20 \mathrm{~s}$. The recording duration corresponds 
to a frequency resolution of $\Delta_{f}=1 / 20=0.05 \mathrm{~Hz}$. In Figure 3, the spectrum of the vibration signal measured on the motor is given. Three tests for three load levels were performed. As expected, we find, in the top figure a component at the rotational frequency. In addition, a component is present at twice the power frequency. In the following, we show that the sensitivity of the motor models imposes strong constraints on this FFT in particular on the number of samples to be considered for the estimation.
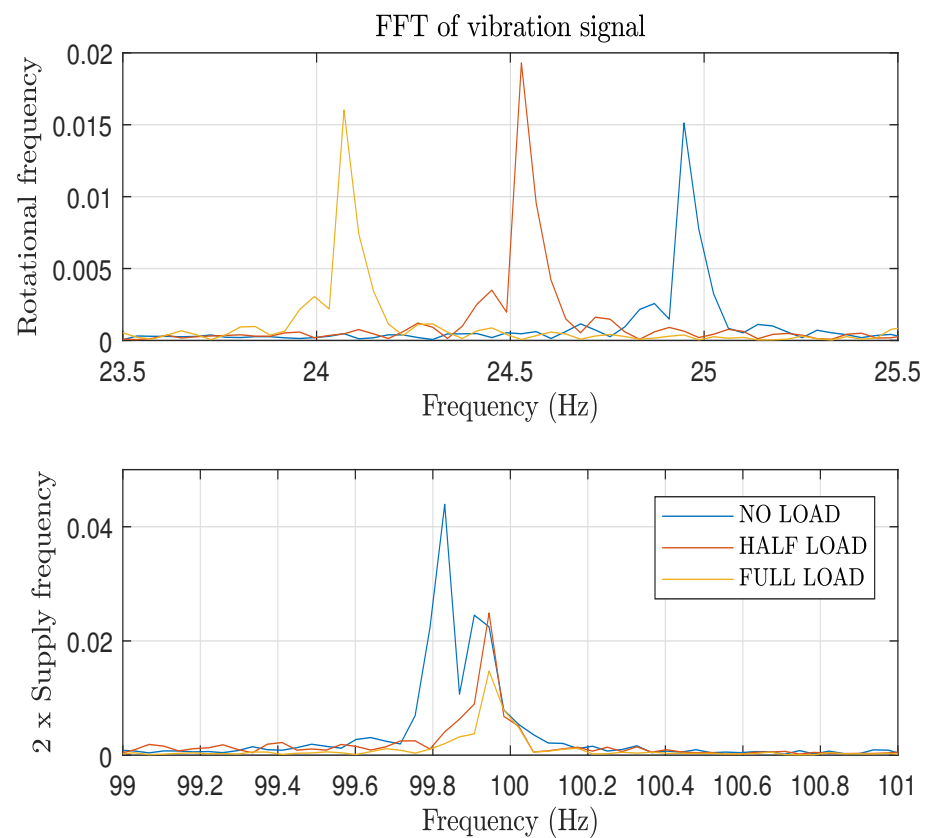

Figure 3. FFT of vibration signals.

\subsection{Motor Models}

\subsubsection{Model $P_{m}=f\left(N_{m}\right)$}

In an industrial context, these characteristic is generally not available. The first model $P_{m}=$ $f\left(N_{m}\right)$ can be deduced from the nameplate. In the motor operating area, this characteristic can be approximated by a quadratic function. The coefficients are calculated simply from the rated speed and rated power indicated on the nameplate. The model can be derived from the followings equations:

$$
\begin{aligned}
T_{m}=g\left(N_{m}\right) & =a+b N_{m}, \\
P_{m} & =T_{m} \frac{\pi N_{m}}{30},
\end{aligned}
$$

with $T_{m}$ the mechanical torque, and $a, b$ two constants to be determined from the nameplate with

$$
\begin{array}{r}
a=\frac{T_{m_{N}}}{f_{r m_{N}}-f_{e_{N}}}, \\
b=-a f_{e_{N}} .
\end{array}
$$

For our motor and from information in Figure 2, the power model is:

$$
P_{m}=f\left(N_{m}\right)=\frac{\pi a}{1800} N_{m}^{2}+\frac{\pi b}{30} N_{m}=-4.93910^{-2} N_{m}^{2}+65.894 N_{m}
$$

The characteristic $T_{m}=g\left(N_{m}\right)$ is a quasi-vertical straight line, which indicates a high sensitivity of the calculated mechanical power with respect to variations in the speed. As a consequence, the model $P_{m}=f\left(N_{m}\right)$ is very sensitive to small variations in speed. If the information from the nameplate is 
inaccurate, the power estimate may be incorrect. It is possible to refine the model by estimating for example the equivalent model of the motor [21].

2.3.2. Model $\eta=h\left(P_{m}\right)$

In commercial documentation, efficiency information are given for four operating points: $P_{m_{N}}$, $0.75 P_{m_{N}}, 0.5 P_{m_{N}}$ and $0.25 P_{m_{N}}$. From documentation of several manufacturers, we have grouped motors identical to the motor closed to our. The criteria for comparison are nominal mechanical power, nominal speed and efficiency class (IE). Four average efficiency values for the four operating points were estimated (Table 1) from about 40 motors comparable to our.

Table 1. Manufacturer data.

\begin{tabular}{ccccc}
\hline $\boldsymbol{P}_{\boldsymbol{m}} / \boldsymbol{P}_{\boldsymbol{m}_{N}}$ & $\mathbf{2 5 \%}$ & $\mathbf{5 0 \%}$ & $\mathbf{7 5 \%}$ & $\mathbf{1 0 0 \%}$ \\
\hline$\eta$ & 0.708 & 0.807 & 0.827 & 0.822 \\
\hline
\end{tabular}

From Table 1, a characteristic $\eta=h\left(P_{m}\right)$ can be approximated by a polynomial models of 3rd order using a Least Square method (LS) (8):

$$
\eta_{1}=h\left(P_{m}\right)=0.476+1.266\left(\frac{P_{m}}{P_{m_{N}}}\right)-1.496\left(\frac{P_{m}}{P_{m_{N}}}\right)^{2}+0.576\left(\frac{P_{m}}{P_{m_{N}}}\right)^{3}
$$

This curve is plotted in green on Figure 4. For $P_{m}=0$ the estimated efficiency is equal to $50 \%$ which is not realistic. So we add a fifth point of coordinates [0; 0]. The L.S algorithm gives the Equation (9).

$$
\eta_{2}=h\left(P_{m}\right)=0.0068+4.0993\left(\frac{P_{m}}{P_{m_{N}}}\right)-6.392\left(\frac{P_{m}}{P_{m_{N}}}\right)^{2}+3.1146\left(\frac{P_{m}}{P_{m_{N}}}\right)^{3}
$$

In these conditions, the polynomial model has two points of inflection. It is found the efficiency characteristic is moving away from the real behavior of the motor (Figure 4).

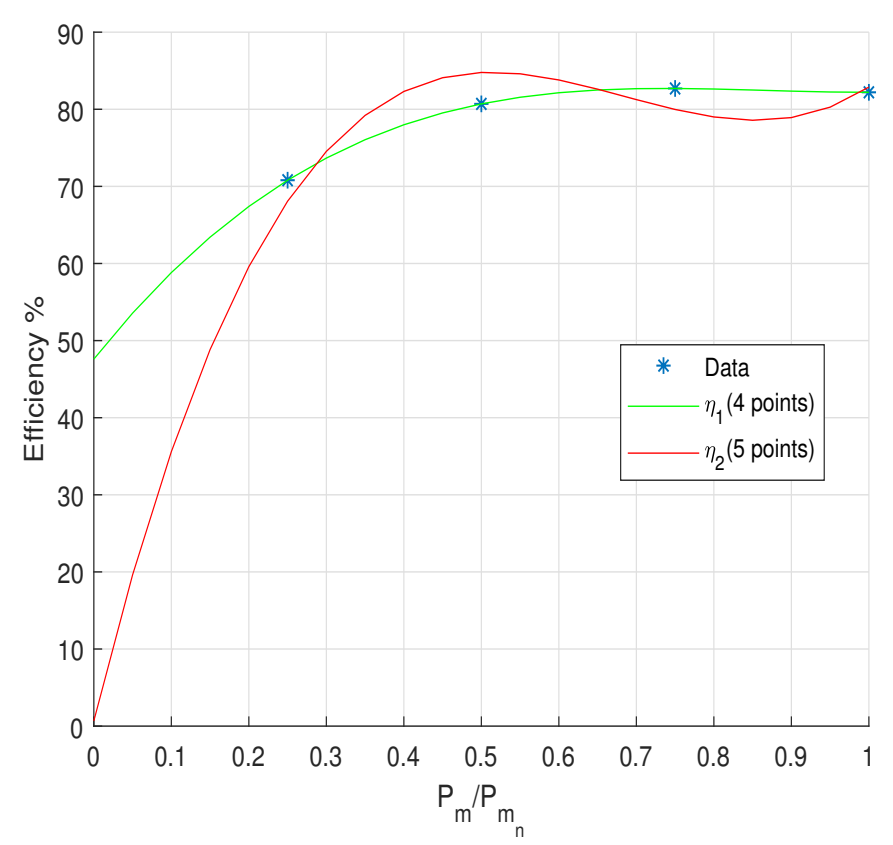

Figure 4. Polynomial Model of 3rd Order of efficiency (4 and 5 points). 
An alternative way is proposed taking into account the motor losses. From the manufacturer data, we calculate the normalized losses according to the efficiency by following the relation:

$$
\begin{aligned}
& \eta=\frac{P_{m}}{P_{m}+\text { Losses }}=\frac{P_{m} / P_{m_{N}}}{P_{m} / P_{m_{N}}+\text { Losses } / P_{m_{N}}} \\
& \eta=\frac{x}{x+\text { Losses } / P_{m_{N}}} \quad \text { with } x=\frac{P_{m}}{P_{m_{N}}} \\
& \text { Normalized losses: } \quad \frac{\text { Losses }}{P_{m_{N}}}=x \frac{1-\eta}{\eta}
\end{aligned}
$$

Table 2. Manufacturer Data and normalized losses.

\begin{tabular}{ccccc}
\hline$x=\boldsymbol{P}_{\boldsymbol{m}} / \boldsymbol{P}_{\boldsymbol{m}_{N}}$ & $\mathbf{2 5 \%}$ & $\mathbf{5 0} \%$ & $\mathbf{7 5 \%}$ & $\mathbf{1 0 0} \%$ \\
\hline$\eta$ & 0.708 & 0.807 & 0.827 & 0.822 \\
Losses $/ P_{m_{N}}$ & 0.1031 & 0.1196 & 0.1567 & 0.2165 \\
\hline
\end{tabular}

We propose a Polynomial model of 3rd order to the standardized losses of the Equation (12). In the least squares sense and with the four points of the Table 2, we determine the four coefficients $p_{0}$, $p_{1}, p_{2}$ and $p_{3}$ of the polynomial model (13).

$$
\begin{gathered}
\frac{\text { Losses }}{P_{m_{n}}}=p_{0}+p_{1} x+p_{2} x^{2}+p_{3} x^{3} \\
\frac{\text { Losses }}{P_{m_{n}}}=0.1059-0.048 x+0.1424 x^{2}+0.0162 x^{3}
\end{gathered}
$$

This polynomial model described in (14) is not enough. It must be considered that the no-load losses are equal to $35 \%$ of the losses under full load according to the assumptions of [22].

$$
\begin{aligned}
& \frac{\text { Losses }_{\text {no load }}}{P_{m_{n}}}=35 \% \times\left(p_{0}+p_{1}+p_{2}+p_{3}\right)=0.07575 \\
& \text { Losses }_{\text {no load }}=0.07575 \times P_{m_{n}}=227.32 \mathrm{~W}
\end{aligned}
$$

Thus, these no-load losses will be useful to us to establish the fifth point as defined in the table below:

The polynomial's coefficients (13) are computed again, in the least squares sense, taking into account the five points of the array Table 3. The losses are defined by the polynomial model described in (15):

$$
\text { Losses }=P_{m_{n}} \times\left(0.0762+0.1317 x-0.1681 x^{2}+0.1771 x^{3}\right)
$$

From the relations (11) and (15), the expression of the efficiency becomes:

$$
\eta=\frac{x}{x+\left(0.0762+0.1317 x-0.1681 x^{2}+0.1771 x^{3}\right)}
$$

Table 3. Manufacturer data with the no-load loss point.

\begin{tabular}{cccccc}
\hline $\boldsymbol{x}=\boldsymbol{P}_{\boldsymbol{m}} / \boldsymbol{P}_{\boldsymbol{m}_{n}}$ & $\mathbf{0 \%}$ & $\mathbf{2 5 \%}$ & $\mathbf{5 0 \%}$ & $\mathbf{7 5 \%}$ & $\mathbf{1 0 0 \%}$ \\
\hline$\eta$ & 0 & 0.708 & 0.807 & 0.827 & 0.822 \\
Losses $/ P_{m_{n}}$ & 0.07575 & 0.1031 & 0.1196 & 0.1567 & 0.2165 \\
\hline
\end{tabular}


Corresponding losses and efficiency curves for our motor are shown on Figure 5.
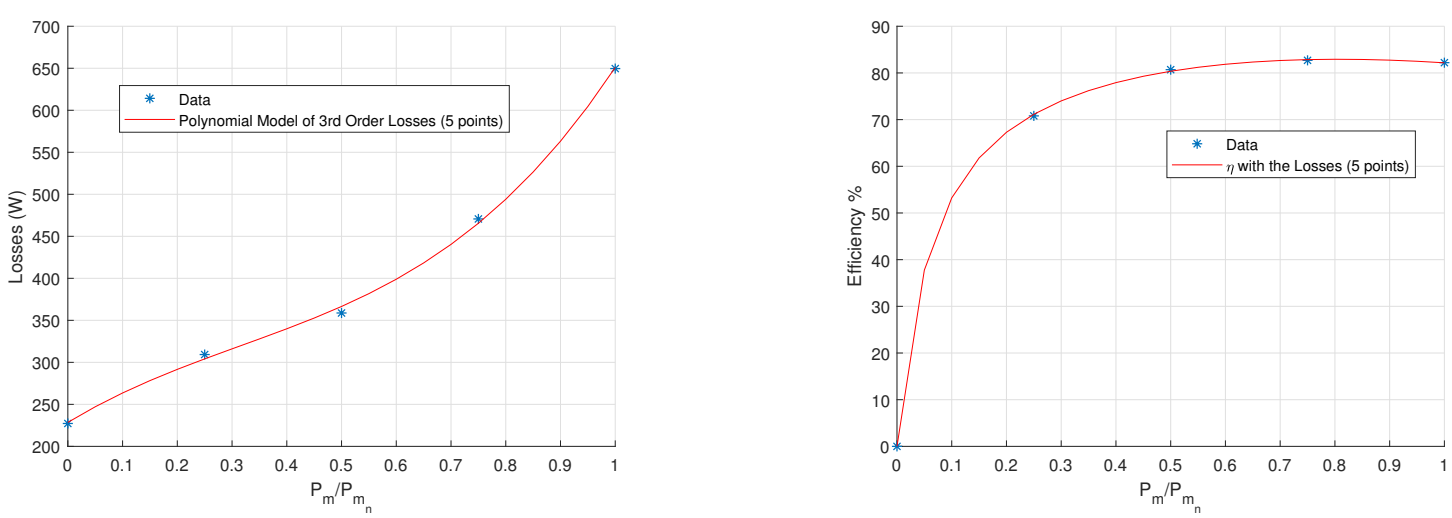

Figure 5. Loss and Efficiency Curves of the Motor Based on the Polynomial Model of 3rd Order Losses.

As mentioned previously, the model (4) is very sensitive to the error on the estimated speed. In the next section, we study the accuracy of the speed estimate to get a good estimate of power and efficiency.

\subsubsection{Sensibility of Motor Models}

Let us consider a permissible error $\Delta \eta(\%)$ on the efficiency estimation. After a brief calculation from models (7) and (16), it is possible to determine the necessary precision on mechanical power $\Delta P_{m}(\%)$ and rotational frequency $\Delta f_{r m}(\mathrm{~Hz})$ estimations. This precision leads to constraints on spectral analysis in terms of resolution. Indeed, consider now a FFT processing from data sampled at the frequency $F_{s}$, the resolution of the FFT is given by:

$$
\Delta_{f}=\frac{F_{s}}{N}=\frac{1}{D^{\prime}}
$$

with $F_{S}$ the sampling frequency $(\mathrm{Hz}), T_{S}=\frac{1}{F_{S}}$ the sampling period (s), $N$ the number of samples and $D=N T_{s}$ the duration of recording (s). The duration $D$ must be chosen so that $\Delta_{f}<\Delta f_{r m}$. Results are shown on Table 4 for the operating point $P_{m}=400 \mathrm{~W}$ which is the worst case because the slope is maximum at this point on the characteristic $\eta=h\left(P_{m}\right)$.

Table 4. Precision on frequency estimation.

\begin{tabular}{cccc}
\hline$\Delta \boldsymbol{\eta}(\%)$ & $\boldsymbol{\Delta} \boldsymbol{P}_{\boldsymbol{m}}(\%)$ & $\boldsymbol{\Delta} \boldsymbol{f}_{\boldsymbol{m}}(\mathrm{Hz})$ & $\boldsymbol{D}(\mathbf{s})$ \\
\hline 10 & $\approx 30$ & $\approx 0.3$ & $>3$ \\
5 & $\approx 20$ & $\approx 0.2$ & $>5$ \\
1 & $\approx 4$ & $\approx 0.04$ & $>25$ \\
\hline
\end{tabular}

It should be noted that when a good estimation is reached, the duration of recording can be prohibitive. In order to obtain a faster estimate, we propose to use another estimation method based on a single-phase PLL.

\section{Online Rotor Speed Estimation}

\subsection{PLL Principle and Improvements}

The PLL used in this paper has been first proposed in [15]. The OSG block provides two signals whose phase shifts differ by 90 degres over the entire frequency spectrum (Figure 6). This result in two quadrature signals supplied to a control loop which provides the instantaneous frequency and phase. The phase detection is built with blocks defined by two filters: 


$$
\left\{\begin{array}{l}
G(s)=\frac{\omega_{R}^{2} s}{s^{2}+2 \omega_{R} s+\omega_{R}^{2}} \\
G^{\prime}(s)=\frac{\omega_{R}^{2}}{s^{2}+2 \omega_{R} s+\omega_{R}^{2}}
\end{array}\right.
$$

- $G_{(s)}$ : Band-pass filter with a central frequency of $\omega_{R}$,

- $G^{\prime}(s)$ : Low-pass filter with a cut-off frequency of $\omega_{R}$.

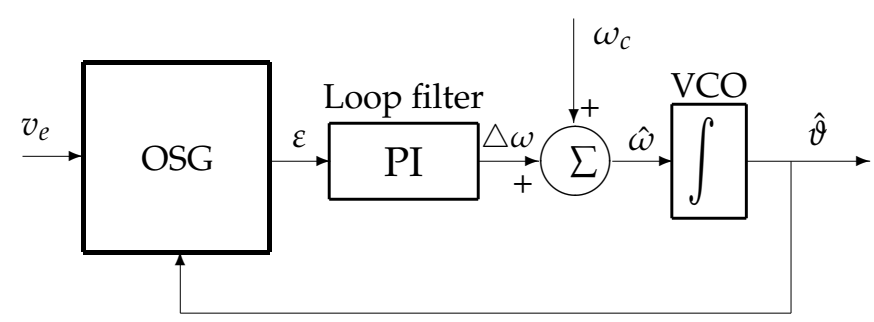

Figure 6. PLL-Basic structure.

We can note that filters (18) provide signals with amplitudes that are not equals. Furthermore, as stated in [15], the gain of the PLL $\left(K_{p d}\right)$, must be adapted as a function of the amplitude of the input signals. In the conventional case of electrical network monitoring, amplitude variations remain limited and this gain is easily tunable. In our case, an accelerometer provides a signal whose amplitude is not predictable and depends on the position of the sensor or on the signal to noise ratio. These amplitude variations result in fluctuations in the estimated frequency and consequently wide variations in the power estimation due to the sensitivity of the motor model. In order to adapt this PLL to our application, we propose to modify the original structure by producing a normalized signals with amplitude \pm 1 , regardless of the sensor used and its position. The normalization is possible if the signals $v_{\alpha}$ and $v_{\beta}$ have the same amplitude, which is not the case with the filters (18). The same output amplitude is obtained by:

- modifying the filter $G(s)$ as:

$$
G(s)=\frac{\omega_{R} s}{s^{2}+2 \omega_{R} s+\omega_{R}^{2}},
$$

- $\quad$ tuning the two filters with the estimated frequency $\hat{\omega}$.

To obtain an adaptive functioning, these filters are simulated using a modified variable state structure shown on Figure 7. The damping coefficient is set to $m=1 / 2$.

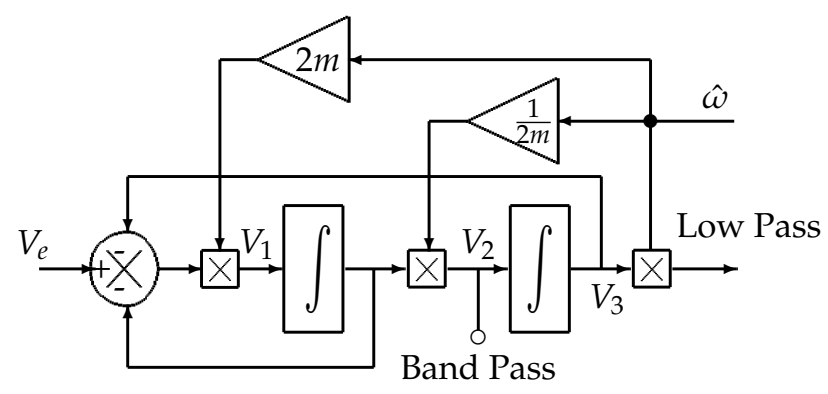

Figure 7. State variable filter.

Now, the outputs of OSG, $v_{\alpha}$ and $v_{\beta}$ may be normalized using the amplitude $\sqrt{\left(v_{\alpha}^{2}+v_{\beta}^{2}\right)}$. Moreover, in our application, the operating range of the motor is limited between its nominal speed 
$\left(f_{r m_{N}}=23.92 \mathrm{~Hz}\right)$ and the frequency of synchronism $\left(f_{e_{N}}=25 \mathrm{~Hz}\right)$. A first second order bandpass filtering is applied to the signal before it is directed to the OGS. The center frequency of this filter is set to the center frequency of the PLL VCO which is at the middle of the operating range $\left(f_{c}=24.46 \mathrm{~Hz}\right)$. The corresponding input block is shown on Figure 8.

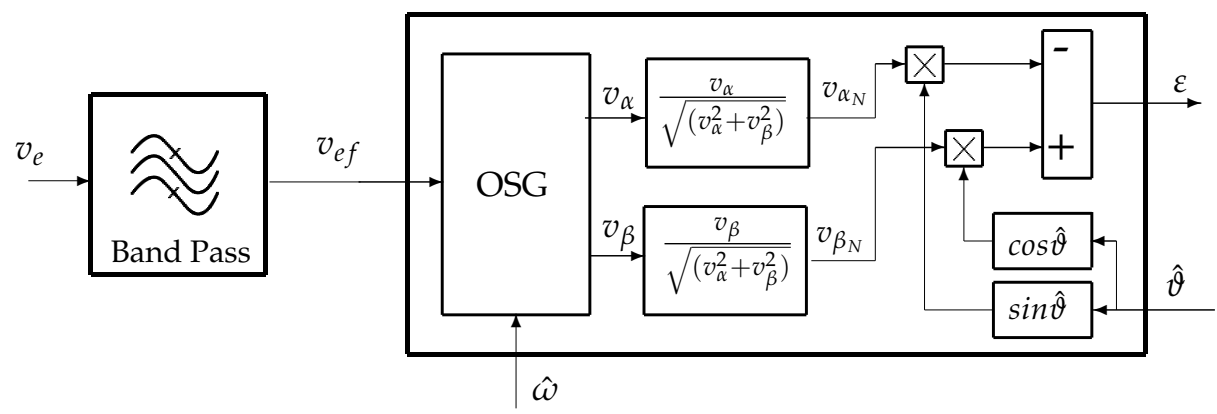

Figure 8. Band Pass and Phase detection.

An integral proportional controller (PI), whose the transfer function is $k_{p}+k_{i} / s$, was placed in the phase locked loop. According to [15], the closed loop transfer function of the system is derived as

$$
H_{c}(s)=\frac{\theta}{\theta_{e}}=\frac{K_{p} K_{p d} s+K_{i} K_{p d}}{s^{2}+K_{p} K_{p d} s+K_{i} K_{p d}}
$$

When the PLL is nearly blocked, $\omega=\omega_{e}$. The phase detector gain is $k_{p d}=\omega_{e} / 4$ with $f_{e}=\omega_{e} / 2 \pi$ of the signal frequency $v_{e}$. If the input signal $v_{e f}$ has an amplitude $V_{m_{e f}}, K_{p d}$ must be divided by $V_{m_{e f}}$. The standard representation of a second order system can be rewritten as

$$
H_{c}(s)=\frac{2 \xi \omega_{n} s+\omega_{n}^{2}}{s^{2}+2 \xi \omega_{n} s+\omega_{n}^{2}}
$$

The performance of second-order systems is normally measured by the step response, which settling time can be approximately calculated by [23]

$$
t_{s} \approx \frac{4.6}{\xi \omega_{n}}
$$

$\xi=0.707$ is the best choice to achieve both a fast rise time and the settling time $t_{s}$ should be no more than $100 \mathrm{~ms}$. We are able to determine $\omega_{n}$. So there is another way to calculate $\omega_{n}$. According to [23], $\omega_{n}$ also depends on the noise bandwidth of the PLL with the relation

$$
B_{L}=\int_{a}^{b}|H(2 j \pi F)|^{2} d F \approx \frac{\omega_{n}}{8 \xi}\left(1+4 \tilde{\zeta}^{2}\right)
$$

In order to eliminate harmonics effectively, we choose $B_{L} \approx 25 \times 2 \pi(\mathrm{rad} / \mathrm{s})$. So we can calculated $\omega_{n}$ by

$$
\omega_{n}=\frac{8 \xi}{\left(1+4 \xi^{2}\right)} B_{L}=\frac{4 \sqrt{2}}{3} B_{L} \approx 296.2 \mathrm{rad} / \mathrm{s}
$$

and the resulting settling time can be calculated as $t_{s}=21.96 \mathrm{~ms}$ following as Equation (22).

The frequency of the input signal $v_{e}$ can vary between $24 \mathrm{~Hz}$ and $25 \mathrm{~Hz}$, the central frequency of filters $\omega_{R}$ is initially placed in the center of this variation $\omega_{R}=24.5 \times 2 \pi(\mathrm{rad} / \mathrm{s})$. When the PLL is nearly blocked, $\omega=\omega_{e} \approx \omega_{R}$ and $K_{p d}=\omega_{e} / 4 \approx 38.48 \mathrm{rad} / \mathrm{s}$. The parameters of the PI controller can be calculated as 


$$
\left\{\begin{array}{l}
K_{p}=2 \xi \omega_{n} / K_{p d} \\
K_{i}=\omega_{n}^{2} / K_{p d}
\end{array}\right.
$$

\subsection{Simulations}

In order to obtain two normalized signals $v_{\alpha_{N}}$ and $v_{\beta_{N}}$, the OSG block must provide two signals $v_{\alpha}$ and $v_{\beta}$ in quadrature with the same amplitude. This is the case only if the frequency of the input signal is equal to the cutoff frequency of the filters $G(s)$ and $G^{\prime}(s)$. In the opposite case, oscillations appear around the frequency estimated by the PLL. A simulation is proposed to show the interest of the adaptation of the OSG filters. A sinusoidal signal of variable frequency is sent to the input of the PLL. The frequency varies according to a ramp of $2 \mathrm{~s}$ between $24 \mathrm{~Hz}$ and $25 \mathrm{~Hz}$. The center frequency of the PLL is fixed at $f_{c}=24.46 \mathrm{~Hz}$ (middle of the oprating range of our motor). In Figure 9a, the OSG frequency $f_{R}$ is set at $f_{R}=f_{c}$. The PLL is locked on the input frequency but the estimated frequency oscillates in steady state. In Figure 9b, the OSG frequency is $f_{R}=24 \mathrm{~Hz}$. The oscillations disappear around $\hat{f}=24 \mathrm{~Hz}$ but remain around $25 \mathrm{~Hz}$. Results are similar for $f_{R}=25 \mathrm{~Hz}$ on Figure 9c. In Figure 9d, the OSG frequency is adapted with the PLL output. The oscillations disappear over the entire range of variation of the input frequency.
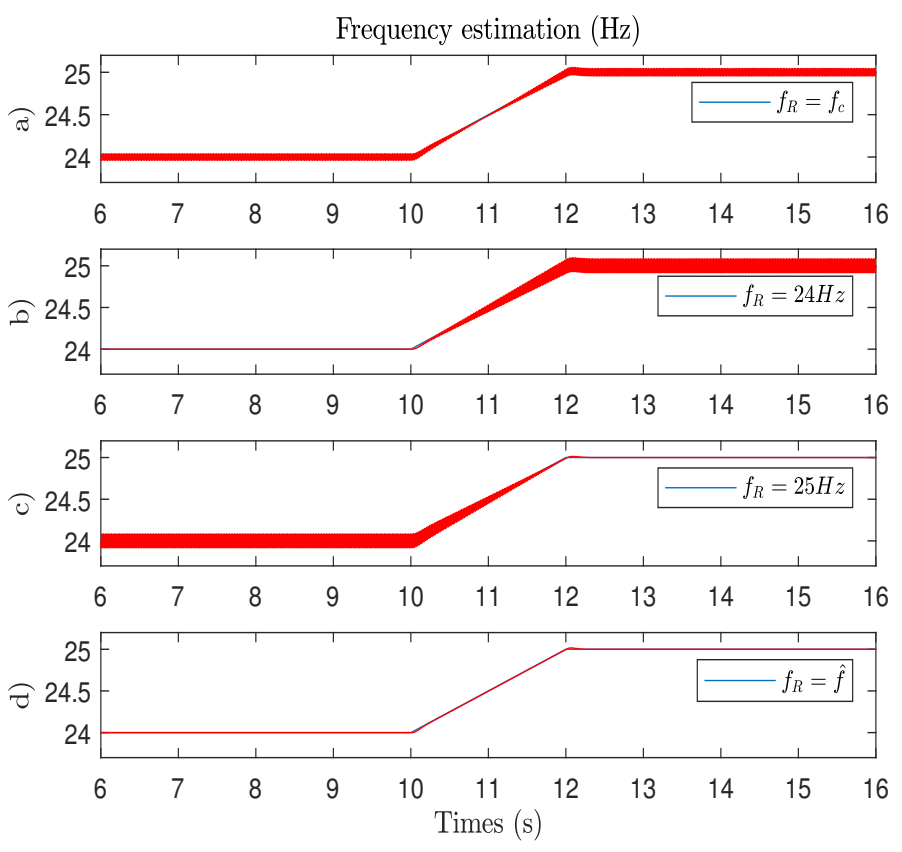

Figure 9. Estimated Frequency: sinusoidal signal with ramp frequency from $24 \mathrm{~Hz}$ to $25 \mathrm{~Hz}$.

(a) $f_{R}=f_{c}$, (b) $f_{R}=24 \mathrm{~Hz}$, (c) $f_{R}=25 \mathrm{~Hz}$, (d) $f_{R}=\hat{f}$.

\section{Experimental Results}

\subsection{Constant Speed}

The first test is carried out at constant speed for three operating point (full load, half load, no load). The estimated frequency obtained with the PLL is compared with a FFT estimation for a recording time of $20 \mathrm{~s}$ corresponding to a resolution $\Delta_{f}=0.05 \mathrm{~Hz}$. This FFT estimation is considered as the reference. On the left of Figure 10, we can see the signals at different stages of estimation. The output band-pass provides a quasi sinusoidal signal with amplitude modulation. As expected, the OSG with normalization generates two orthogonal sinus compared with the PLL ouput. The PLL is correctly locked resulting in phase comparator error near to zero. On the right, all the motor quantities calculated with procedure resumed on Figure 1 are shown. Table 5 shows estimations of motor quantities and 
the relative error in \% for the electrical power $P e$ for three operating points. The PLL provides the same accuracy that the FFT but in real-time. Therefore, it is not necessary to have a long recording to achieve the desired precision. This property will be used for variable speed functioning in the next section. Accuracy is less than $10 \%$ for the entire operating range of the motor. Several sources of error may explain this lake of accuracy. First, the information on the rating plate may be inaccurate. In addition, these information are valid only if the motor has reached its rated operating temperature (approximately one hour of operation at nominal load for a motor of this power). We respected this constraint during our tests. Another influence factor is the supply voltage $(V)$. In our case it was higher than the nominal value, $\left(V=136 \mathrm{~V}\right.$ for un nominal value $\left.V_{N}=132.8 \mathrm{~V}\right)$. In theory, the efficiency should be corrected by a factor $K=\left(\frac{V}{V_{N}}\right)^{2}$. This correction has not been applied here because we wanted to be in conditions where only the vibratory measurement is available. Finally, the efficiency model (16) comes from a database of motors similar to the motor studied. This can be an additional source of error.
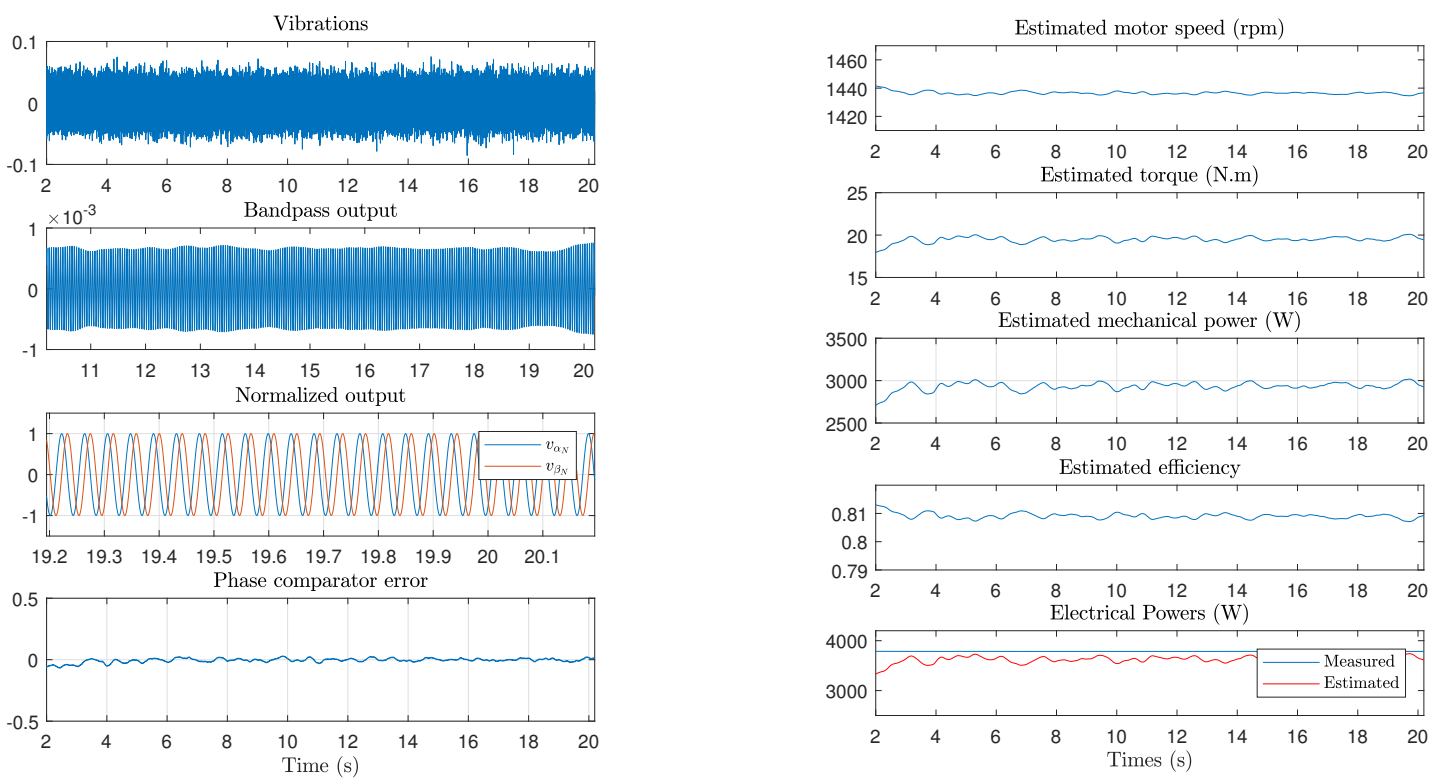

Figure 10. Experimental test at constant speed and full load operating point.

\subsection{Variable Speed}

On the left of Figure 11, we show variable speed tests from no load to full load. The estimation of the electrical power in real-time is validated. The estimated power correctly follows the measured power. The proposed method therefore allows dynamic monitoring of the motor consumption. It is clear that it is limited to slow variations of the operating point because Equations (7) and (16) correspond to static model. In order to show the contribution of our PLL on a vibratory signal, we have tested the PLL proposed in [15] in its original form:

- OSG are not tuned with the estimated frequency provided by the PLL,

- OSG at the PLL output is maintained,

- $\quad$ signals are not normalized,

- the P.I the setting is the same as our. 
Table 5. Experimental test at constant speed, nominal values: $N_{N}=1435 \mathrm{rpm}, P m_{N}=3000 \mathrm{~W}$, $T m_{N}=19.96$ N.m, $\eta_{N}=81.5 \%, P e_{N}=3681 \mathrm{~W}$.

\begin{tabular}{cccc}
\hline & No Load & Half Load & Full Load \\
\hline$\widehat{N}_{F F T}(\mathrm{rpm})$ & 1496.7 & 1464.6 & 1435.5 \\
$\widehat{N}_{P L L}(\mathrm{rpm})$ & 1497.1 & 1464.1 & 1436.4 \\
$\epsilon_{N}(\%)$ & 0.0272 & 0.0404 & 0.064 \\
$\widehat{T}_{m}(\mathrm{~N} . \mathrm{m})$ & 0.893 & 11.04 & 19.53 \\
$\widehat{P}_{m}(\mathrm{~W})$ & 140 & 1692.7 & 2938.4 \\
$\widehat{\eta}(\%)$ & 35.1 & 80 & 80.89 \\
$\widehat{P}_{e}(\mathrm{~W})$ & 392.77 & 2115.6 & 3623.3 \\
$P_{e}(\mathrm{~W})$ & 375.46 & 2319 & 3785.3 \\
$\epsilon_{P_{e}}(\%)$ & 4.61 & 8.8 & 4 \\
\hline
\end{tabular}

Results are shown on the right of Figure 11. As expected, the PLL proposed in [15] does not work in its original form for a non-stationnary vibration signal. Indeed, the input OSG provides quadrature signals of different and non-normalized amplitudes. These signals are compared to the signals provided by the OSG at the output of the PLL which are of amplitude \pm 1 . The phase error is never zero and the loop can not be stabilized. Moreover, in the case of an input variable frequency, the output amplitudes of input OSG vary because the gains of the filters vary according to the frequency. It is clear that the same results would be obtained with other types of PLL designed for monitoring the frequency of an electrical network. The adaptation of the OSG filters as well as the normalization of the signals make it possible to solve these problems and to propose an exploitable system for non-stationary signals with variable amplitude.
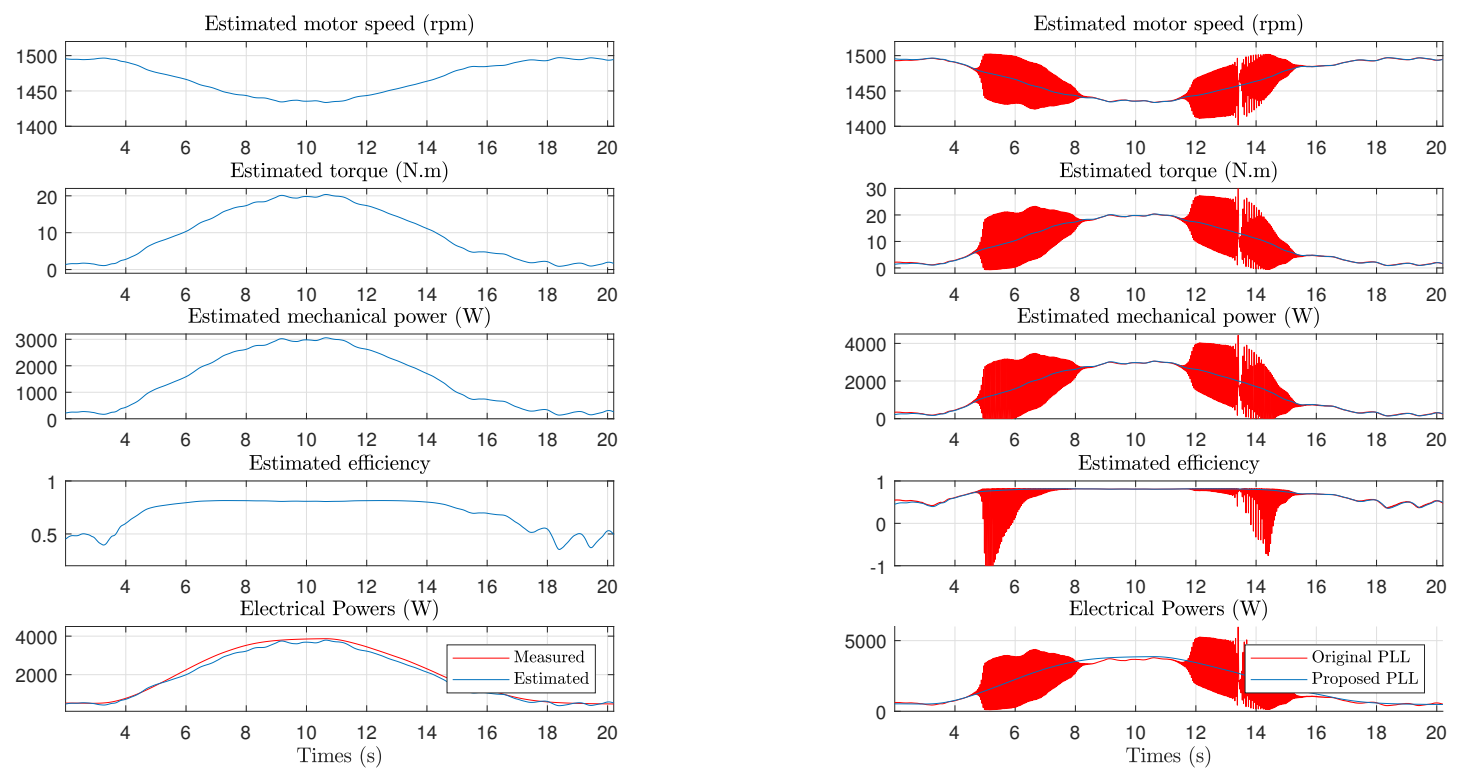

Figure 11. Experimental test at variable speed. Proposed PLL (left), orignal PLL (rigth).

\section{Conclusions}

In this paper, we have shown the use of a PLL to estimate the rotational frequency of a variable speed asynchronous motor from a vibration measurement. This estimate is then used to calculate the electrical power consumed. The vibratory sensor can additionally provide the operating time of the motor, and thus, the energy consumed. This system performs an energy measurement without any electrical measurement. PLLs are often used in estimating the frequency of the electrical network which is a simpler case. Indeed, in this case, the frequency and the amplitude of the measured signal 
do not vary to a great extentand the signal-to-noise ratio is high. We have shown that to use this type of PLL on a vibratory signal of variable frequency and amplitude, with a low SNR, improvements are necessary. This is what we proposed using an adaptive OSG and a normalization of signals (frequency and phase) provided by the PLL. All the motor quantities are estimated only from nameplate and motor databases. Future work will probably concern the adaptation of the models to variations in motor temperature and power supply via a variable speed drive.

Author Contributions: T.D., E.E., L.R. and S.C. proposed the main idea of the paper; E.E. developed vibration analysis and PLL adaptation. T.D. developed motor models and database. L.R. and S.C. implemented simulation verification, analyses and experimental tests; The paper was written by T.D. and E.E., and was revised by L.R., S.C. All the authors were involved in preparing the final version of this manuscript. Besides, this whole work is supervised by T.D. and E.E.

Funding: French ANR agency for the funding of LabCom IONESCO Project ANR-17-LCV2-0004.

Acknowledgments: The authors would like to thank the French ANR agency for the funding of LabCom IONESCO Project ANR-17-LCV2-0004.

Conflicts of Interest: The authors declare no conflict of interest.

\section{References}

1. Zhang, H.; Bradley, K.; Zanchetta, P. A None-Intrusive Load and Efficiency Evaluation Method for In-Service Motors Using Vibration Tests with an Accelerometer. In Proceedings of the IEEE Industry Applications Society Annual Meeting, Edmonton, AB, Canada, 5-9 Octobert 2010.

2. Dlamini, V.; Naidoo, R.; Manyage, M. A non-intrusive method for estimating motor efficiency using vibration signature analysis. Electr. Power Energy Syst. 2010, 45, 384-390. [CrossRef]

3. Lin, H.; Ding, K. A new method for measuring engine rotational speed based on the vibration and discrete spectrum correction technique. Measurement 2013, 46, 2056-2064. [CrossRef]

4. Netsanet, S.; Zhang, J.; Zheng, D. Bagged Decision Trees Based Scheme of Microgrid Protection Using Windowed Fast Fourier and Wavelet Transforms. Electronics 2018, 7, 61. [CrossRef]

5. Freijedo, F.D.; Doval-Gandoy, J.; Lopez, O.; Fernandez-Comesana, P.; MartinezGions, C. A signal-processing adaptive algorithm for selective current harmonic cancellation in active power filters. IEEE Trans. Ind. Electron. 2009, 56, 2829-2840. [CrossRef]

6. Golestan, S.; Ramezani, M.; Guerrero, J.M. DQ-frame cascaded delayed signal cancellation-based PLL: Analysis, design, and comparison with moving average filter-based PLL. IEEE Trans. Power Electron. 2015, 30, 1618-1632. [CrossRef]

7. $\mathrm{Yu}, \mathrm{B}$. An Improved Frequency Measurement Method from the Digital PLL Structure for Single-Phase Grid-Connected PV Applications. Electronics 2018, 7, 150. [CrossRef]

8. Golestan, S.; Monfared, M.; Freijedo, F.D.; Guerrero, J.M. Design and tuning of a modified power-based PLL for single-phase grid-connected power conditioning systems. IEEE Trans. Power Electron. 2012, 27, 3639-3650. [CrossRef]

9. Thacker, T.; Boroyevich, D.; Burgos, R.; Wang, F. Phase-locked loop noise reduction via phase detector implementation for single-phase systems. IEEE Trans. Ind. Electron. 2011, 58, 2482-2490. [CrossRef]

10. Han, Y.; Luo, M.; Zhao, X.; Guerrero, J.M.; Xu, L. Comparative Performance Evaluation of Orthogonal-Signal-Generators-Based Single-Phase PLL Algorithms-A Survey. IEEE Trans. Power Electron. 2016, 31, 3932-3944. [CrossRef]

11. Cao, Y.; Yu, J.; Xu, Y.; Li, Y.; Yu, J. An Efficient Phase-Locked Loop for Distorted Three-Phase Systems. Energies 2017, 10, 280. [CrossRef]

12. Rodriguez, P.; Teodorescu, P.; Candela, I.; Timbus, A.V.; Liserre, M.; Blaabjerg, F. New positive-sequence voltage detector for grid synchronization of power converters under faulty grid conditions. In Proceedings of the 37th IEEE Annual Power Electronics Specialists Conference, Jeju, Korea, 18-22 June 2006.

13. Ciobotaru, M.; Teodorescu, R.; Blaabjerg, F. A new single-phase PLL structure based on second order generalized integrator. In Proceedings of the 37th IEEE Annual Power Electronics Specialists Conference, Jeju, Korea, 18-22 June 2006. 
14. Golestan, S.; Monfared, M.; Freijedo, F.D.; Guerrero, J.M. Dynamics assessment of advanced single-phase PLL structures. IEEE Trans. Ind. Electron. 2013, 30, 2167-2177. [CrossRef]

15. Guan, Q.; Zhang, Y.; Kang, Y.; Guerrero, Y.M. Single-Phase Phase-Locked Loop Based on Derivative Elements. IEEE Trans. Power Electron. 2016, 32, 4411-4420. [CrossRef]

16. Boglietti, A.; Cavagnino, A.; Lazzari, M.; Pastorelli, M. International Standards for the Induction Motor Efficiency Evaluation: A Critical Analysis of the Stray-Load Loss Determination. IEEE Trans. Ind. Appl. 2004, 40, 1294-1301. [CrossRef]

17. Lu, B.; Habetler, T.G.; Harley, R.G. A survey of efficiency-estimation methods for in-service induction motors. IEEE Trans. Ind. Appl. 2006, 42, 924-933.

18. Al-Badri, M.; Pillay, P.; Angers, P. A Novel In Situ Efficiency Estimation Algorithm for Three-Phase IM Using GA IEEE Method F1 Calculations and Pretested Motor Data. IEEE Trans. Energy Convers. 2015, 30, 1092-1102. [CrossRef]

19. Salomon, C.P.; Santana, W.C.; Lambert-Torres, G.; Borges da Silva, L.; Bonaldi, E.; de Oliveira, L. Comparison among Methods for Induction Motor Low-Intrusive Efficiency Evaluation Including a New AGT Approach with a Modified Stator Resistance. Energies 2018, 11, 691. [CrossRef]

20. Wu, D.; Pekarek, S.D. Using Mechanical Vibration to Estimate Rotor Speed in Induction Motor Drives. In Proceedings of the 2007 IEEE Power Electronics Specialists Conference, Orlando, FL, USA, 17-21 June 2007.

21. Al-Jufout, S.A.; Al-rousan, W.H.; Wang, C. Optimization of Induction Motor Equivalent Circuit Parameter Estimation Based on Manufacturer's Data. Energies 2018, 11, 1792. [CrossRef]

22. Szychta, L.; Figura, R. Analysis of efficiency characteristics of squirrel-cage induction motor for pump applications. In Proceedings of the 2012 International Conference on Electrical Machines, Marseille, France, 2-5 September 2012.

23. Gardner, F.M. Phase Lock Techniques; Wiley: New York, NY, USA, 1979.

(C) 2019 by the authors. Licensee MDPI, Basel, Switzerland. This article is an open access article distributed under the terms and conditions of the Creative Commons Attribution (CC BY) license (http://creativecommons.org/licenses/by/4.0/). 